\title{
Beyond the \\ Virtual Intracranial Stenting Challenge 2007: non-Newtonian and flow pulsatility effects
}

\author{
Marco Cavazzuti $^{\mathrm{a}}$, Mark Atherton ${ }^{*}$, , Michael Collins $^{\mathrm{b}}$, Giovanni Barozzi ${ }^{\mathrm{a}}$ \\ ${ }^{a}$ Dipartimento di Ingegneria Meccanica e Civile, Università degli Studi \\ di Modena e Reggio Emilia, via Vignolese 905, 41125 Modena, Italy \\ ${ }^{b}$ School of Engineering and Design, Brunel University, West London, UB8 3PH, UK
}

\begin{abstract}
The Virtual Intracranial Stenting Challenge 2007 (VISC'07) is becoming a standard test case in computational minimally-invasive cerebrovascular intervention. Following views expressed in the literature and consistent with the recommendations of a report, the effects of non-Newtonian viscosity and pulsatile flow are reported. Three models of stented cerebral aneurysms, originating from VISC'07 are meshed and the flow characteristics simulated using commercial Computational Fluid Dynamics (CFD) software. We conclude that non-Newtonian and pulsatile effects are important to include in order to discriminate more effectively between stent designs.

Key words: cerebral aneurysm, visc'07, stent
\end{abstract}

\section{Introduction}

The work presented here uses benchmark models from the Virtual Intracranial Stenting Challenge (VISC 2007), an international initiative to assess the effectiveness of state-of-the-art numerical modelling of blood flow in stented cerebral aneurysms. The results submitted to VISC'07 by six simulation teams (Radaelli et al. 2008) highlight the desirability of considering the effects of non-Newtonian viscosity and flow pulsatility in future work for purposes of clinical relevance, both of which were included in our studies briefly reported here.

*corresponding author

Email addresses: mark. atherton@brunel.ac.uk (Mark Atherton), +44(0) 1895 274000 (Mark Atherton)

Preprint submitted to Elsevier

May 4, 2010 


\section{Methods}

The Fluent code was used for the simulations. The following boundary conditions were set: uniform velocity with $2.36 \frac{\mathrm{g}}{\mathrm{s}}$ mass flow rate at the inlet, zero gauge pressure on the outlet and no slip walls, as set by VISC'07.

A steady-state laminar solver was used with second order upwind momentum discretization and SIMPLE pressure-velocity coupling. The fluid flowing in the artery was initially defined as water (as requested by VISC'07), and later it was changed to blood with density $(\rho) 1060 \frac{\mathrm{kg}}{\mathrm{m}^{3}}$ and viscosity $(\mu) 4 \mathrm{cP}$.

Since a Newtonian model is prone to underestimate the WSS in a CFD analysis at lower velocity gradients (Chen et al. 2006, Lee and Steinman 2006, Gijsen et al. 1999a,b), it was decided to investigate a non-Newtonian formulation as well. The Fluent power-law option for dynamic viscosity (Fluent 2006) was used, as no one model is universally accepted and this is a valid option at lower shear rates (Johnson et al. 2004, Shibeshi and Collins 2005):

$$
\mu_{\min }<\mu=k \cdot \dot{\gamma}^{n-1}<\mu_{\max }
$$

where $\mu$ is the dynamic viscosity in $\frac{\mathrm{kg}}{\mathrm{ms}}, k$ is the consistency index whose value is $0.0161 \frac{\mathrm{kgs}^{n-2}}{\mathrm{~m}}, n$ the power-law index is 0.63 , and $\dot{\gamma}$ is the shear rate in $\mathrm{s}^{-1}$ (Owen et al. 2005). $\mu_{\min }$ and $\mu_{\max }$ are lower and upper limits of the power-law function and were set to $10^{-5} \frac{\mathrm{kg}}{\mathrm{ms}}$ and $1 \frac{\mathrm{kg}}{\mathrm{ms}}$ respectively.

In order to address the inclusion of pulsatility (c.f. Radaelli et al. 2008 and others), unsteady simulations were configured for the unstented and the three stented cases. The inlet waveform for a basilar artery (Ford et al. 2008) was slightly modified as follows: the mean flow rate was set to the steadystate value of $2.36 \frac{\mathrm{g}}{\mathrm{s}}$ as specified by VISC'07 and implemented in Fluent as a uniform velocity profile of $0.179 \frac{\mathrm{m}}{\mathrm{s}}$; the pulse rate was set to 70 beats per minute; and the waveform was slightly smoothed in order to reduce the number of time steps needed to represent the whole cycle.

A standard grid independency procedure on the stented aneurysm models was carried out, and a suitable meshing of 2.30 million elements meshes was selected.

\section{Results}

For brevity, we focus the results on two regions of interest. (i) The aneurysm Neck Section, which corresponds to the minimum section area of the aneurysm and is comparable with that of cut-plane P2 in Radaelli 
Table 1: Comparison between unstented and stented cases for steady-state flow. Percentages refer to difference between stented case and unstented artery.

\begin{tabular}{|l||c||c||c|}
\hline Case & $\begin{array}{c}\text { Mass Flow (g/s) } \\
\text { through Neck Section }\end{array}$ & $\begin{array}{c}\text { Average Dynamic Viscosity } \\
(\mathrm{cP}=\mathrm{mPa} \text { s)in Segment x }\end{array}$ & $\begin{array}{c}\text { Average WSS (Pa) } \\
\text { in Segment x }\end{array}$ \\
\hline \hline Unstented artery & 0.3809 & 7.142 & 2.057 \\
non-Newtonian & 0.3391 & 4.000 & 1.091 \\
\hline \hline Stent 1 & $-11.0 \%$ & $-44.0 \%$ & $-46.9 \%$ \\
Newtonian & 0.3427 & 4.000 & 1.054 \\
\hline Stent 2 & $-10.0 \%$ & $-44.0 \%$ & $-48.8 \%$ \\
Newtonian & 0.2738 & 4.000 & 0.960 \\
\hline Stent 3 & $-28.1 \%$ & $-44.0 \%$ & $-53.3 \%$ \\
Newtonian & 0.3268 & 8.003 & 1.296 \\
\hline \hline Stent 1 & $-14.2 \%$ & $+12.1 \%$ & $-37.0 \%$ \\
non-Newtonian & 0.3320 & 7.938 & -37.282 \\
\hline Stent 2 & $-12.8 \%$ & $+11.1 \%$ & 1.195 \\
non-Newtonian & 0.2785 & 8.555 & $-41.9 \%$ \\
\hline Stent 3 & $-26.9 \%$ & $+19.8 \%$ & \\
non-Newtonian & \multicolumn{3}{|c||}{$\%$} \\
\hline
\end{tabular}

et al. 2008. (ii) Segment $\mathrm{X}$, the first $\frac{1}{3}$ aneurysm volume immediately after the Neck Section. Results are given in terms of mass flow rate and average Wall Shear Stress (WSS).

\subsection{Newtonian vs. non-Newtonian}

Referring to the table of results for steady-state (Table 1), the nonNewtonian case significantly increases dynamic viscosity and slightly modifies mass flow rate in the lower aneurysm area resulting in a much higher average wss. The Newtonian models overestimate mass flow rate by about $3 \%$ for Stent 1 and Stent 2 and underestimate by just under $2 \%$ for Stent 3 , whereas the average wss in the Newtonian models is consistently underestimated by $15-20 \%$.

The results do not contradict Gijsen et al. (1999a,b), who, using both experiments and simulations, essentially state the importance of non-Newtonian (shear-thinning) blood modelling since it alters significantly the velocity profiles. As a non-Newtonian model produces higher fluid viscosities in the aneurysm region (where the shear rate is low) compared to the Newtonian 


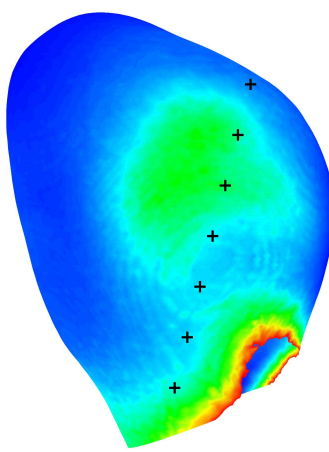

(a)

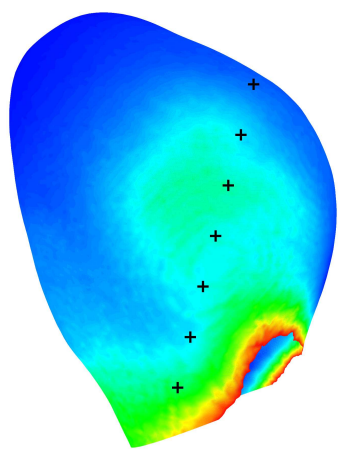

(b)

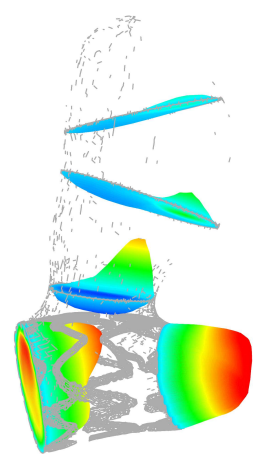

(c)

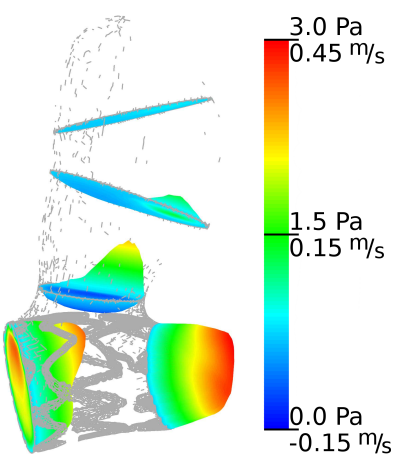

(d)

Figure 1: WSS and velocity profiles in aneurysm region for stent 3 steady simulation: Newtonian versus non-Newtonian: (a) Wss, Newtonian case; (b) Wss, non-Newtonian case; (c) velocity profiles, Newtonian case; (d) velocity profiles, non-Newtonian case

\subsection{Pulsatile flow}

In Figure 2 the mass flow rate entering the aneurysm (i.e. crossing the Neck Section) is shown. The pulse cycle in the main artery is also shown scaled to $\frac{1}{5}$-th of its amplitude.

The mass flow rate entering the aneurysm in the unstented case at time $0 \mathrm{~s}$ is equal to $0.3488 \frac{\mathrm{g}}{\mathrm{s}}$ ( $15 \%$ of the mass flow rate in the main artery). This ratio remains in the range from $10 \%$ to $20 \%$ for all the cases investigated 3 and for most of the pulse cycle, except when the mass flow rate in the artery 4 drops to its minimum.

The presence of the stent reduces the mass flow rate in the aneurysm 6 region and also promotes small changes in the phase between the mass flow 97 rate in the main artery and in the aneurysm. In fact, the main pulse cycle is 


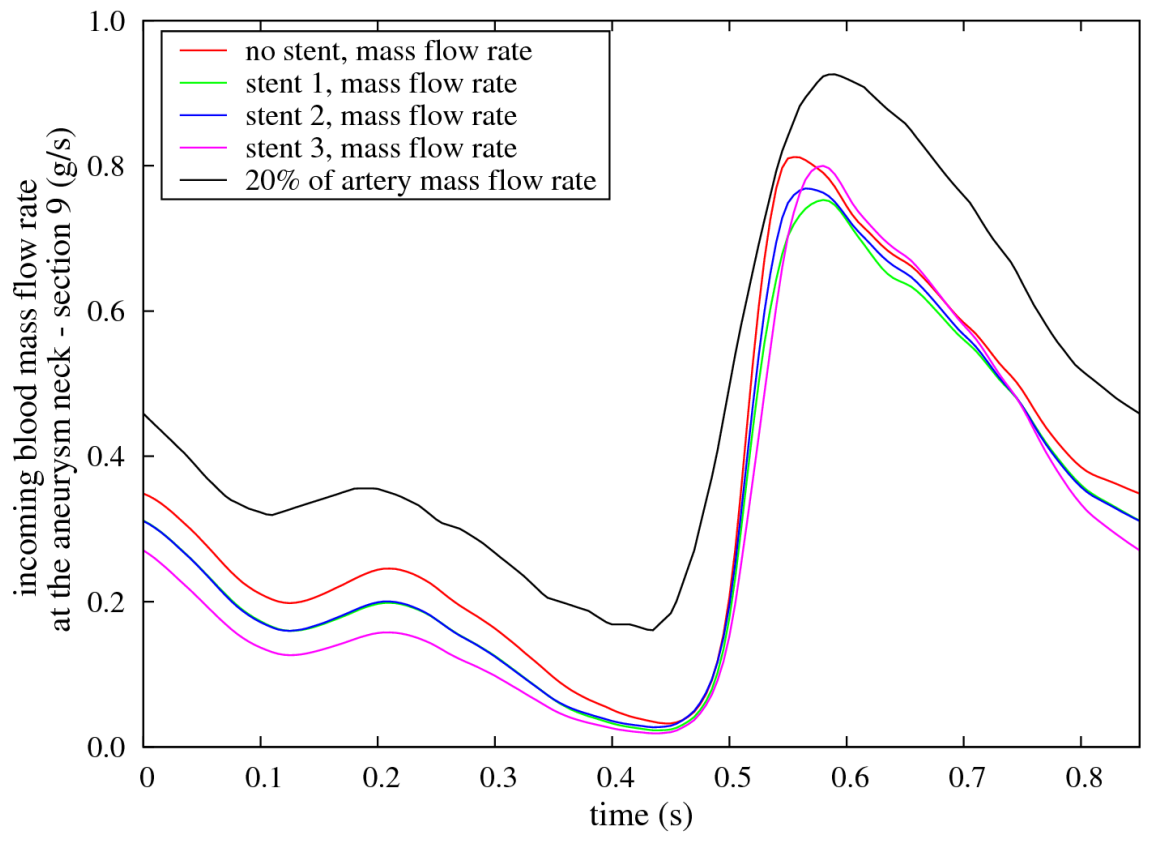

Figure 2: Mass flow through Section 9

reflected in the aneurysm mass flow rate with a minimum phase delay from 0 to $0.5 \mathrm{~s}$. However, the maximum peak in the aneurysm mass flow rate, which is found at $\approx 0.57 \mathrm{~s}$ for each of the four simulations, anticipates the corresponding peak in the main artery, which is found at $0.59 \mathrm{~s}$. The change in the phase shift, even if small, together with the change in mass flow ratio along the cycle suggest the relevance of adopting unsteady simulations for better accuracy.

The third stent is particularly efficient in reducing the mass flow rate entering the aneurysm, in particular in the first half of the pulse cycle with reductions ranging from $20 \%$ to $50 \%$ when compared to the unstented artery. The third stent also shows better performance, when compared with the other two stents, for more than $75 \%$ of the pulse cycle.

\subsection{Evaluating three stents from VISC'07}

Considering the non-Newtonian blood model (see Table 1), the mass flow rates of stent 1 and stent 2 are not so different from each other. Stent 2 has a higher mass flow rate through the Neck Section ( $0.3320 \frac{\mathrm{g}}{\mathrm{s}}$ equal to a $12.8 \%$ reduction in the mass flow rate compared to the unstented case). Stent 3 


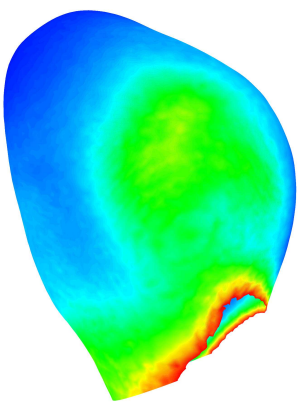

(a) Unstented

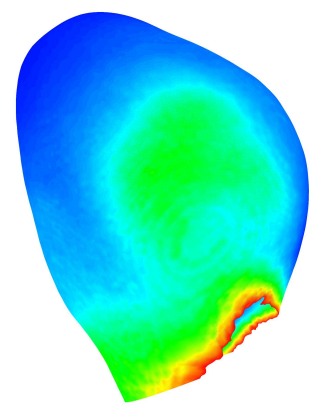

(b) Stent 1

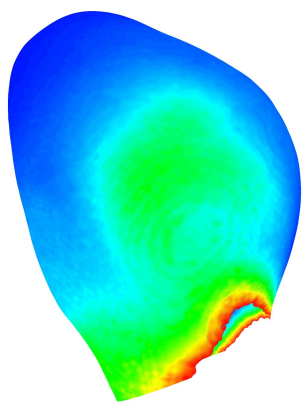

(c) Stent 2

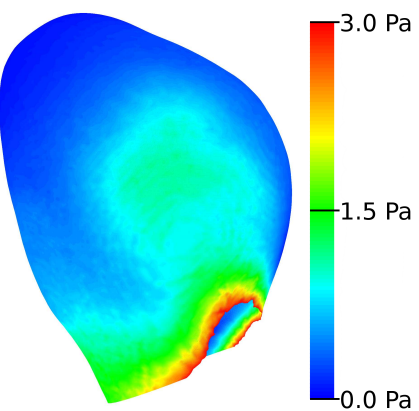

(d) Stent 3

Figure 3: WSS on surface of aneurysm for steady non-Newtonian simulations

appears to be much more effective in reducing the mass flow rate in the aneurysm since the blood crossing the Neck Section in this case amounts to $0.2785 \frac{\mathrm{g}}{\mathrm{s}}$ (a $26.9 \%$ reduction).

From these initial considerations stent 3 is expected to be a better clinical performer than the other two.

Comparing the wss in the unstented case with the three stented models (Figure 3 and Table 1), the use of a stent appears to be very effective in reducing the wss.

\section{Conclusions}

The inclusion of non-Newtonian and pulsatile effects in the VISC'07 models are shown to be important. Stent 3 emerges as the best design, which is consistent with the results published in the literature.

\section{Conflict of interest statement}

The authors have no financial or personal relationships with other people or organisations that could inappropriately influence (bias) their work.

\section{Acknowledgements}

We thank Dr Matthieu De Beule from the Institute Biomedical Technology, Ghent University, Belgium for his generous advice on how to implement the Magics software. Also, our thanks to Dr Peter Flynn, Consultant Radiologist, Victoria Hospital, Belfast Trust, for his helpful comments on clinical aspects of the paper. 


\section{References}

Chen, J., Lu, X-Y., Wang, W., 2006, Non-Newtonian effects of blood flow on hemodynamics in distal vascular graft anastomoses. J. Biomech. 39 (11), 1983-1995.

Fluent 6.3 User Guide, 2006, section 8.4.5.

Ford, M.D., Niklov, H.N., Milner, J.S., Lownie, S.P., DeMont, E.M., Kalata, W., Loth, F., Holdsworth, D.W., Steinman, D.A., 2008. PIV-measured versus CFD-predicted flow dynamics in anatomically realistic cerebral aneurysm models. J. Biomech. Eng. 130 (2), 021015 (9 pages).

Gijsen, F.J.H., van de Vosse, F.N., Janssen, J.D., 1999a. The influence of the non-Newtonian properties of blood on the flow in larger arteries: steady flow in a carotid bifurcation model. J. Biomech. 32 (6), 601-608.

Gijsen, F.J.H., Allanic, E., van de Vosse, F.N., Janssen, J.D., 1999b. The influence of the non-Newtonian properties of blood on the flow in larger arteries: unsteady flow in a 903 curved tube. J. Biomech. 32 (6), 705713.

Johnson, B.M., Johnson, P.R., Corney, S., Kilpatrick, D., 2004. NonNewtonian blood flow in human right coronary arteries: steady state simulations. J. Biomech. 37 (5), 709-713.

Lee, S.-W., Steinman, D.A., 2006. On the relative importance of rheology for image-based CFD models of the carotid bifurcation. J. Biomech. 39 (1), S283.

Owen, I., Gray, J., Escudier, M., Poole, R., 2005. The importance of the nonNewtonian characteristics of blood in flow modelling studies. Abstracts of Second Physiological Flow Network meeting, University of Edinburgh, 26-27 September 2005.

Radaelli, A.G., Augsburger, L., Cebral, J.R., Ohta, M., Rufenacht, D.A., Balossino, R., Benndorf, G., Hose, D.R., Marzo, A., Metcalfe, R., Mortier, P., Mut, F., Reymond, P., Socci, L., Verhegghe, B., Frangi, A.F., 2008. Reproducibility of haemodynamical simulations in a subjectspecific stented aneurysm model - A report on the Virtual Intracranial Stenting Challenge 2007. J. Biomech. 41 (10), 2069-2081. 
Shibeshi, S.S., Collins, W.E., 2005. The Rheology of Blood Flow in a Branched Arterial System. Applied Rheology 15 (6), 398-405.

170 VISC 2007 The 1st Virtual Intracranial Stenting Challenge

171 http://www.cilab.upf.edu/visc06 (accessed 29 January 2009) 